Journal for ImmunoTherapy of Cancer

\section{Treatment of recurrent mucosal melanoma of the oral cavity with topical imiquimod and pembrolizumab achieves complete histopathologic remission}

To cite: Satish T, Khan S, Levin M, et al. Treatment of recurrent mucosal melanoma of the oral cavity with topical imiquimod and pembrolizumab achieves complete histopathologic remission. Journal for ImmunoTherapy of Cancer 2021;9:e001219. doi:10.1136/jitc-2020-001219

Accepted 10 July 2021

Check for updates

(c) Author(s) (or their employer(s)) 2021. Re-use permitted under CC BY-NC. No commercial re-use. See rights and permissions. Published by BMJ.

${ }^{1}$ Columbia University Vagelos College of Physicians and Surgeons, New York, New York, USA

${ }^{2}$ Department of Medicine, Division of Hematology/ Oncology, Columbia University Irving Medical Center, New York, New York, USA

${ }^{3}$ Cell IDx, San Diego, California, USA

${ }^{4}$ Division of Oral and Maxillofacial Pathology, Columbia University Irving Medical Center, New York, New York, USA

Correspondence to

Dr Angela J Yoon;

ajk55@cumc.columbia.edu

\section{ABSTRACT}

Mucosal melanomas constitute a subtype of melanoma with less effective treatments than cutaneous melanomas. We present a case of oral mucosal melanoma that recurred despite multiple resections and adjuvant temozolomide. Treatment with topical imiquimod combined with pembrolizumab achieved remission. A 56-year-old woman presented with a pigmented mass on her left anterior hard palate. Biopsy revealed malignant melanoma. The patient had resection with neck dissection with 3 months of adjuvant temozolomide due to positive margins. Malignant melanoma involving the hard palate recurred 1 year later requiring additional resection. Two years later, two additional pigmented lesions were found; further resections were deferred due to expected morbidity. Following 6 weeks of topical imiquimod treatment, the lesions shrunk significantly. Adjuvant pembrolizumab was added and complete histopathologic remission was observed in 6 months. The patient remained in remission for 4 years before new melanoma in situ was diagnosed, requiring five additional months of imiquimod. As of April 2021, there is no clinical evidence of melanoma. There are limited reports of oral melanoma treated with topical imiquimod. Here, imiquimod administered in combination with pembrolizumab achieved complete pathologic response.

\section{BACKGROUND}

Mucosal melanoma is a rare subtype of melanoma, accounting for $1.3 \%$ of all melanoma cases. ${ }^{1}$ Mucosal melanomas are more aggressive and difficult-to-detect due to their site of development. ${ }^{2}$ The standard-of-care for oral mucosal melanoma is resection. Surgery can carry significant morbidity, and despite complete resection, recurrence is common. Therapies targeting melanoma-specific driver mutations and immunologic checkpoints display lower response rates in mucosal melanomas compared with cutaneous melanomas, prompting the consideration of other strategies. ${ }^{4}$ Toll-like receptor (TLR) agonists have shown promise in the treatment of cutaneous malignancies including melanoma. ${ }^{5}$ However, reports of successful treatment of mucosal melanoma with imiquimod are limited. Here, we report the case of recurrent intraoral melanoma successfully treated with imiquimod.

\section{CASE REPORT}

A 52-year-old woman presented to her head and neck surgeon (ENT) in 2008 with a wellcircumscribed, pigmented lesion of her left lingual gingiva/anterior hard palate. The clinical impression was focal melanosis due to local irritation and observation was recommended. Four years later, the pigmented lesion evolved into multifocal lesion involving both the right (tooth \#2 region) and left (teeth \#9, \#11 and \#12 region) anterior hard palate. Biopsy revealed melanoma in situ. Subsequently, the patient underwent resection of the anterior hard palate with neck dissection in 2012, which revealed malignant melanoma with positive (melanoma in situ) margins. The cervical lymph nodes were negative for melanoma. Mutation analysis revealed wild-type BRAF, KIT and NRAS. A positron emission tomography/CT showed no evidence of distant metastasis. Due to positive surgical margins, radiotherapy was considered. However, to avoid the morbidity associated with irradiation, a decision was made to administer adjuvant chemotherapy (temozolomide) for 3 months.

One year later, in 2014, a biopsy of a new pigmented lesion on the anterior hard palate extending both to the right and left of midline revealed melanoma in situ. The patient underwent another surgical resection of the hard palate. Histology revealed melanoma in situ, again with positive anterior margins. A second resection was performed with clear margins.

In 2016, two pigmented lesions were found in the left anterior hard palate and labial 


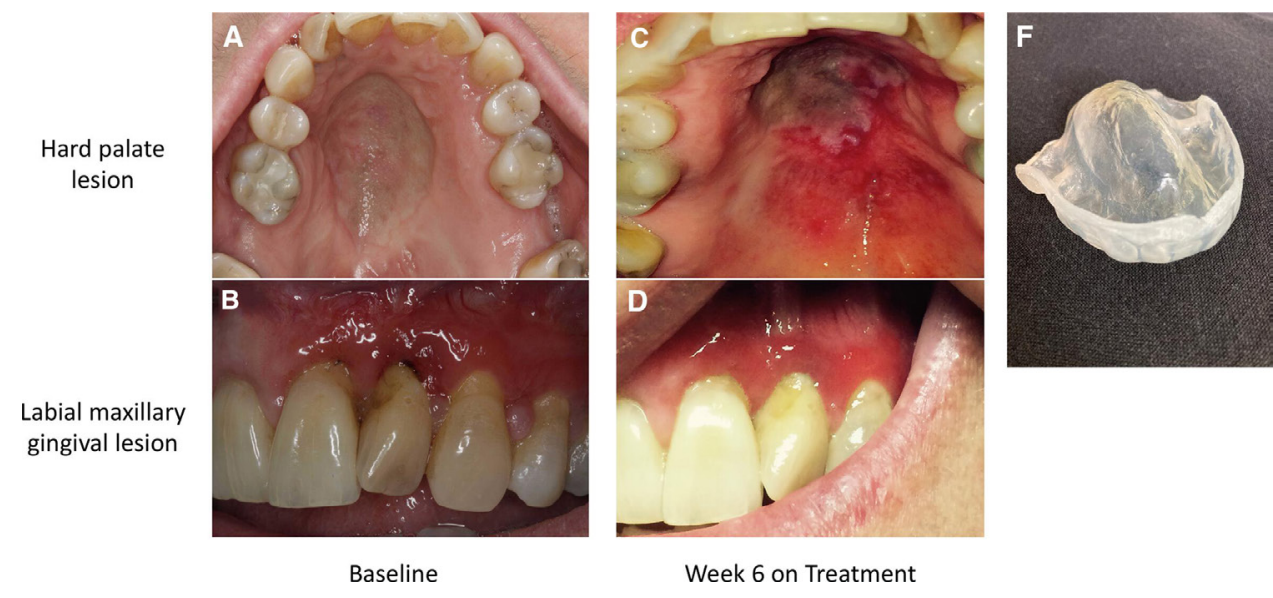

Figure 1 At baseline, pigmented lesions diagnosed as recurrent malignant melanoma are visible on the hard palate $(\mathrm{A})$ and left maxillary gingiva (B). After 6 weeks of single-agent imiquimod therapy, there is a visible reduction in the size of the palatal (C) and gingival lesions (D). (F) The custom tray for imiquimod application. Photos courtesy of Susanna M Goggin, DMD.

gingiva of tooth \#10. An incisional biopsy of one of the lesions revealed atypical intraepithelial lentiginous melanocytic proliferation. Subsequently, an excisional biopsy was performed showing melanoma in situ extending to the lateral margins. An excisional biopsy of the second lesion was negative for melanoma. Given the non-invasive lesions and morbidity associated with multiple surgical resections, observation was recommended.

Shortly thereafter, the patient presented with a new pigmented mass of the anterior hard palate (figure 1A) as well as pigmentation of the labial gingiva of tooth \#10 (figure 1B). An excisional biopsy of the anterior hard palatal lesion revealed malignant melanoma (figure 2A and B) with melanoma in situ extending to the lateral margins. Given her extensive surgical history and high expected morbidity, further resections were deferred.

The locally recurrent multifocal lesion was highly suggestive of field cancerization. The decision was made to initiate topical imiquimod $5 \%$ cream, an

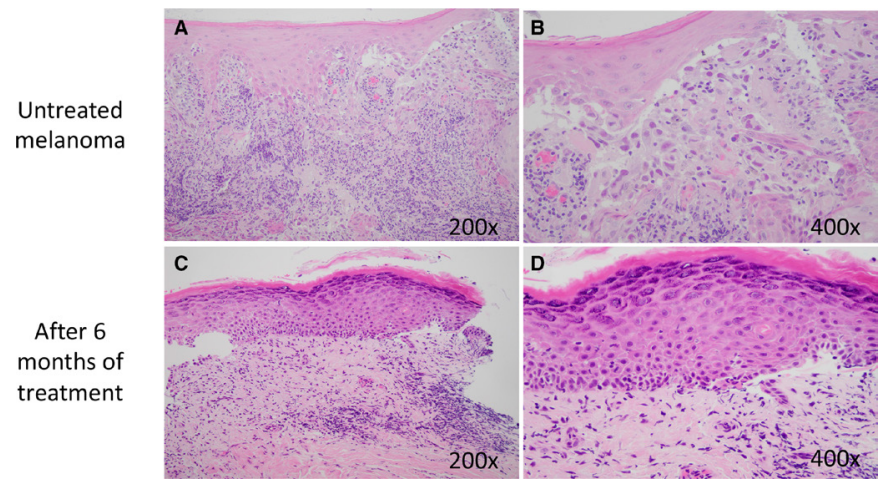

Figure 2 Photomicrographs of the untreated lesion (A) show recurrent malignant melanoma arising from surface epithelium directly invading into the underlying lamina propria. Higher power (B) shows tumor cells with nuclear pleomorphism and melanin granules within the cytoplasm. After 6 months of treatment, histology shows surface epithelium with hyperkeratosis and underlying chronic inflammatory infiltrate $(\mathrm{C})$. There is no evidence of residual melanoma at higher magnification (D). immunomodulatory agent that has been Food and Drug Administration-approved for treatment of cutaneous cancerized fields, precancerous lesions, and carcinomas. Topical imiquimod was applied every other day to the pigmented areas of the hard palate and labial gingiva (October 2016). A custom application tray was fabricated to ensure adequate exposure of the tumor to imiquimod (figure $1 \mathrm{~F}$ ). Biweekly evaluation was performed. The patient initially tolerated the imiquimod well with no adverse events. After 6 weeks of single-agent imiquimod therapy, there was evidence of inflammation in the hard palate lesion (figure 1C) and significant clinical response in the pigmented labial gingival lesion (figure 1D). Due to the uncertain stage of her recurrent disease (residual melanoma in situ that may have progressed into malignant melanoma), we discussed the potential benefit for checkpoint therapy including its potential to improve outcomes in patients with metastatic disease. The patient subsequently was started on pembrolizumab $2 \mathrm{mg}$ / $\mathrm{kg}$ every 3 weeks. She tolerated both imiquimod and pembrolizumab well, experiencing only constipation, fatigue, and arthralgias.

After 2 months of imiquimod therapy, the patient reported irritation and bleeding of the hard palate. Treatment was reduced to once every 3 days. After 6 months of imiquimod and pembrolizumab therapy, repeat biopsy of the left hard palate/gingiva showed no melanoma (figure 2). Given the complete histologic response, topical imiquimod and pembrolizumab were discontinued. Surveillance examinations every 3 months and CT of the neck/chest/abdomen/pelvis every 6 months was done; as of April 2020, scans remained negative.

In August 2020, three new pigmented lesions involving the left anterior hard palate and another lesion involving the palatal gingiva of tooth \#15 were detected (figure 3A and B). An excisional biopsy revealed melanoma in situ of the palate with negative margins and melanosis of the gingiva. Despite negative margins, imiquimod treatment was re-initiated every other day to the affected areas as a preventive measure. After 4 weeks of treatment, the 

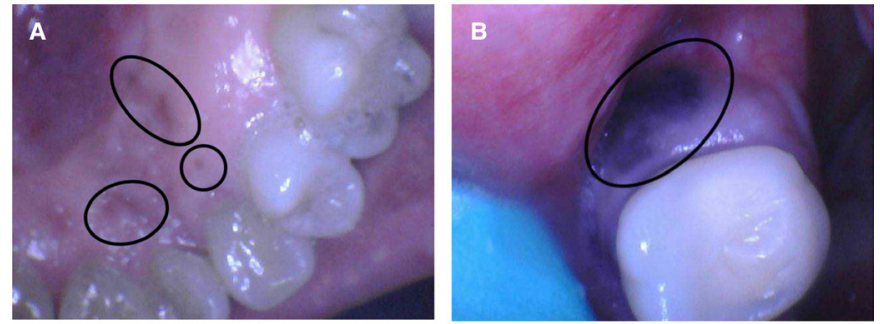

C

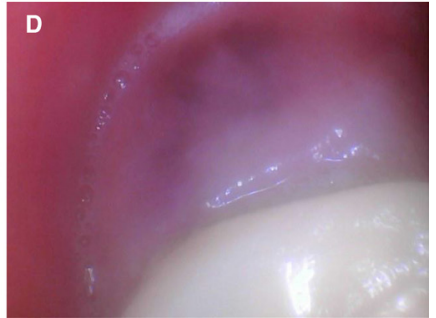

Figure 3 Recurrent pigmented lesions diagnosed as melanoma in situ are visible in the right anterior hard palate (A) and focal melanosis of the gingiva of tooth \#15 (B). After the excisional biopsy with negative margins and prophylactic treatment with topical imiquimod, no recurrent lesion is evident in the anterior palate $(\mathrm{C})$ and minimal residual melanosis is overserved in gingiva (D). Photos courtesy of Susanna M Goggin, DMD.

patient experienced significant discomfort near the palatal gingival lesion, dysgeusia, and flu-like symptoms including fatigue and chills. These symptoms abated following a reduction in treatment to every third day. After 12 weeks of treatment, the patient resumed every other day application with improved tolerance. At week 15 , treatment was held due to a recurrence of pain and bleeding in the gingival treatment area which selfresolved. After 20 weeks of treatment, the patient developed an infected molar requiring extraction. Imiquimod
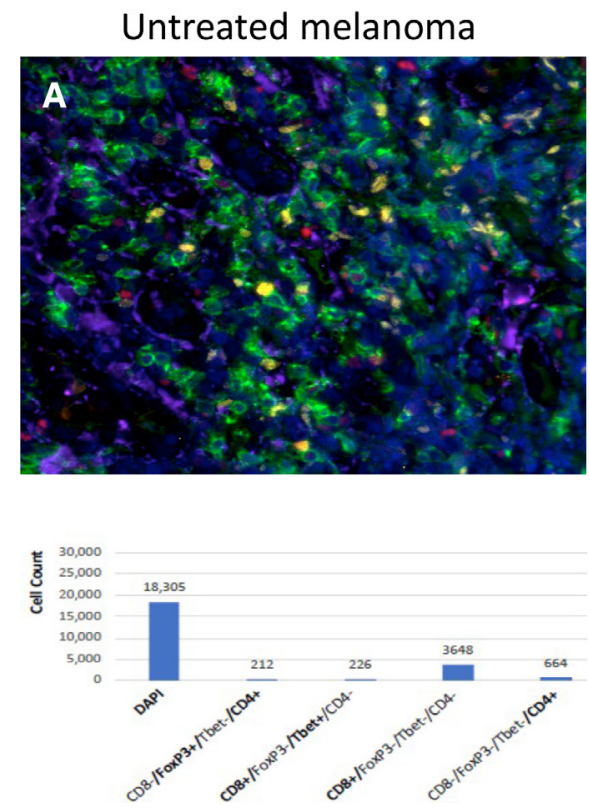

Figure 4 Quantitative multiplex immunofluorescence analysis of immune cell profiles before (A) and after 6 months (B) of topical imiquimod and pembrolizumab therapy. Markers: DAPI (black), CD4 (purple), FoxP3 (yellow), Tbet (red), CD8a (green). The immunomodulatory effect of imiquimod treatment is visible as increased numbers of activated CD4 + Tcells.

was stopped at this time; the residual pigmentation at the gingival margin of tooth \#15 was no longer visible (figure $3 \mathrm{C}$ and $\mathrm{D})$. There is no clinical evidence of recurrent melanoma as of April 2021.

Immune cell profiles in the patient's recurrent melanoma in 2016 and following treatment with topical imiquimod and pembrolizumab were assessed retrospectively using quantitative multiplex immunofluorescence assays (figure 4). The formalin-fixed paraffin-embedded tissue slides were stained with anti-human CD8 (a cytotoxic T-cell marker), FoxP3 (a regulatory T-cell marker), Tbet (a Th1-type helper T-cell marker), CD4 (a helper T-cell marker), and DAPI (a nuclear marker). Stained slides were scanned on the Leica Versa with positive and negative controls and the images were adjusted using Aperio ImageScope (V.12.4.2.5010). Data were analyzed using a macro derived from the Leica Quantitative Algorithm V.1. Inflamed oral mucosa following imiquimod treatment revealed almost a 10 -fold increase in activated helper T-cell population (CD4+/CD8-/FoxP3-/Tbet-) and a $13 \%$ increase in cytotoxic T-cell population (CD8+/ CD4-/FoxP3-/Tbet-) compared with the recurrent melanoma.

\section{DISCUSSION AND CONCLUSIONS}

Mucosal melanoma of the oral mucosa is rare $^{16}$ and challenging to treat due to its aggressive behavior and tendency to manifest asymptomatically in occult locations. $^{2}$ The standard-of-care for oral mucosal melanoma is complete resection, sometimes with the underlying bone, resulting in significant morbidity. Local and distant recurrence following resection is not unusual. ${ }^{3} 7$ Adjuvant radiotherapy improves local control but not overall
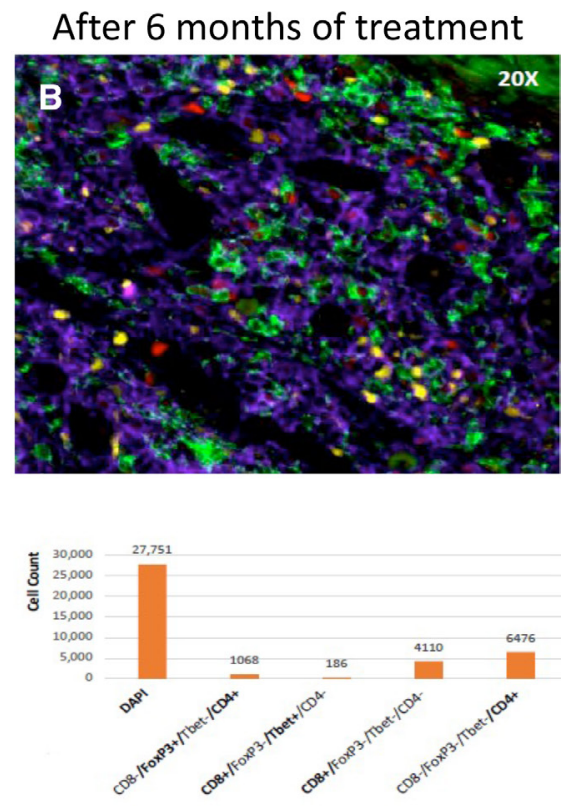
survival. ${ }^{8}$ Five-year survival rate for mucosal melanoma remains low $(20 \%-35 \%)$, highlighting a need for alternative treatments. ${ }^{10}$

For cutaneous melanoma, therapeutic agents targeting common mutations, such as BRAF, have been employed with much success. However, only $15 \%-17 \%$ of mucosal melanomas harbor these mutations. ${ }^{4}$ Immunological checkpoint blockade using antibodies that inhibit the programmed cell death 1 on T-cells has demonstrated modest benefit in mucosal melanoma. One pooled analysis reported an overall response rate (ORR) of 23.3\% in patients receiving nivolumab alone, and an ORR of $37.1 \%$ in patients receiving nivolumab with ipilimumab. ${ }^{11}$ Another retrospective cohort analysis reported a similar ORR of $23 \%$ in mucosal lesions. ${ }^{12}$ While we used the checkpoint inhibitor pembrolizumab, given the aforementioned limited response rates with these agents, the histopathological response observed may be, in part, attributed to the use of imiquimod.

Imiquimod is a TLR agonist demonstrated to have antitumorous effects mediated via initiation of apoptosis and activation of helper T-cells. ${ }^{1314}$ On binding to TLRs, imiquimod leads to activation of nuclear factor- $\mathrm{\kappa B}$, which stimulates the production of pro-inflammatory cytokines (interleukin-6/8) and other components of innate immunity. ${ }^{14}$ The end result is a helper T cell-mediated antitumor response. ${ }^{14-16}$ Moreover, imiquimod increases tumor cell apoptosis by shifting the proapoptotic and antiapoptotic $\mathrm{Bcl}$ factors toward the proapoptotic Bax protein, and also by stimulating the release of mitochondrial cytochrome c into the cytosol, activating caspase- 9 and caspase- $-3 .^{15} 16$

Imiquimod has previously been used to successfully treat cutaneous melanoma in situ. ${ }^{1718}$ We and others have reported patients with oral mucosal melanoma refractory to surgery, chemotherapy, and radiation successfully treated with topical imiquimod for 6 months. ${ }^{19}{ }^{20}$ For our patient, recurrent melanoma was seen only 1year following adjuvant chemotherapy with temozolomide (in 2013) for positive surgical margins. A complete surgical resection with negative margins (in 2014) allowed disease remission of 2 years before recurrence was observed. With application of topical imiquimod (in 2016), the patient was in complete remission for 4 years (until year 2020). Although the value of additional pembrolizumab cannot be evaluated, the patient demonstrated a significant clinical response following 6 weeks of single-agent imiquimod therapy. One of the challenges identified was intraoral application of the medication, requiring frequent in-office appointments for imiquimod application by the physician..$^{20}$ We employed a custom tray to allow home application and prolong the contact of imiquimod with the tumor.

Analysis of FFPE tissues immediately prior to and following treatment with imiquimod and pembrolizumab using multiplex immunofluorescence demonstrated significant shift in immune cell profile. Following application of imiquimod, there was a ninefold increase in activated helper T-cells contributing to antitumorous response. There was also an increase in activated cytotoxic T-cells after treatment (and a decrease in inactive cytotoxic T-cells). Regulatory T-cells (Tregs; CD4 + FoxP3+) in tumor microenvironments regulate other cells of the adaptive immunity, including CD8 + T cells and B-cells, to suppress anticancer immune response, thereby promoting tumor development and progression. ${ }^{21}$ Treg population was detected in the recurrent melanoma tissue. In a nonneoplastic environment, Tregs have an essential role in regulating cells of innate immunity, such as macrophages and neutrophils, that are critical for the maintenance of immune tolerance. ${ }^{22}$ After 6 months of treatment with imiquimod and pembrolizumab, an increased number of Tregs were identified in chronically inflamed mucosa, supporting their role in innate immunity.

In summary, imiquimod therapy can induce antitumorous response by activation of helper T-cells, leading to complete and durable histopathologic remission of melanoma. Topical application allows for maximum effect on the tumor without significant systemic side effects. Hence, $5 \%$ imiquimod cream represents a significant addition to the available immunomodulatory therapies for effective treatment of oral melanoma.

Acknowledgements We would like to acknowledge Susanna M Goggin, DMD, for her clinical photographs of the patient. We would also like to acknowledge Elizabeth Philipone, DMD, for her consultation regarding the pathological specimens in the case.

Contributors TS analyzed and interpreted the patient's clinical data and drafted the manuscript. SK reviewed the patient's clinical data and edited the manuscript. ML generated figure 2 and performed multiplex immunofluorescence analysis. AJY reviewed and interpreted patient data and edited the manuscript. RC obtained the primary clinical data, supervised the manuscript production. All authors read and approved the final manuscript.

Funding This work was supported by the NIH/NIDCR R01DE026801 (AJY) and NIH/ NCI R21CA252441 (AJY, RDC).

Competing interests No, there are no competing interests.

Patient consent for publication Consent obtained directly from patient(s).

Ethics approval Need for approval was waived.

Provenance and peer review Not commissioned; externally peer reviewed.

Open access This is an open access article distributed in accordance with the Creative Commons Attribution Non Commercial (CC BY-NC 4.0) license, which permits others to distribute, remix, adapt, build upon this work non-commercially, and license their derivative works on different terms, provided the original work is properly cited, appropriate credit is given, any changes made indicated, and the use is non-commercial. See http://creativecommons.org/licenses/by-nc/4.0/.

\section{ORCID iD}

Tejus Satish http://orcid.org/0000-0002-9697-4058

\section{REFERENCES}

1 Chang AE, Karnell LH, Menck HR. The National Cancer Data Base report on cutaneous and noncutaneous melanoma: a summary of 84,836 cases from the past decade. the American College of Surgeons Commission on Cancer and the American Cancer Society. Cancer 1998;83:1664-78.

2 Carvajal RD, Spencer SA, Lydiatt W. Mucosal melanoma: a clinically and biologically unique disease entity. J Natl Compr Canc Netw 2012;10:345-56.

3 Wong JH, Cagle LA, Storm FK, et al. Natural history of surgically treated mucosal melanoma. Am J Surg 1987;154:54-7. 
4 Tyrrell H, Payne M. Combatting mucosal melanoma: recent advances and future perspectives. Melanoma Manag 2018;5:MMT11-MMT.

5 Park AJ, Paul J, Chapman MS, et al. Long-term outcomes of melanoma in situ treated with topical 5\% imiquimod cream: a retrospective review. Dermatol Surg 2017;43:1017-22.

6 McLaughlin CC, Wu X-C, Jemal A, et al. Incidence of noncutaneous melanomas in the U.S. Cancer 2005:103:1000-7.

7 Medina JE, Ferlito A, Pellitteri PK, et al. Current management of mucosal melanoma of the head and neck. J Surg Oncol 2003:83:116-22.

8 Temam S, Mamelle G, Marandas P, et al. Postoperative radiotherapy for primary mucosal melanoma of the head and neck. Cancer 2005;103:313-9.

9 Owens JM, Roberts DB, Myers JN. The role of postoperative adjuvant radiation therapy in the treatment of mucosal melanomas of the head and neck region. Arch Otolaryngol Head Neck Surg 2003;129:864-8.

10 Moreno MA, Roberts DB, Kupferman ME, et al. Mucosal melanoma of the nose and paranasal sinuses, a contemporary experience from the M. D. Anderson cancer center. Cancer 2010;116:2215-23.

11 D'Angelo SP, Larkin J, Sosman JA, et al. Efficacy and safety of nivolumab alone or in combination with ipilimumab in patients with mucosal melanoma: a pooled analysis. J Clin Oncol 2017;35:226-35

12 Shoushtari AN, Munhoz RR, Kuk D, et al. The efficacy of anti-PD-1 agents in acral and mucosal melanoma. Cancer 2016;122:3354-62.
13 Burns RP, Ferbel B, Tomai M, et al. The imidazoquinolines, imiquimod and R-848, induce functional, but not phenotypic, maturation of human epidermal Langerhans' cells. Clin Immunol 2000;94:13-23.

14 Stanley MA. Imiquimod and the imidazoquinolones: mechanism of action and therapeutic potential. Clin Exp Dermatol 2002;27:571-7.

15 Navi D, Huntley A. Imiquimod 5 percent cream and the treatment of cutaneous malignancy. Dermatol Online J 2004:10:4.

16 Schön MP, Schön M. Imiquimod: mode of action. Br J Dermatol 2007;157 Suppl 2:8-13.

17 Swetter SM, Chen FW, Kim DD, et al. Imiquimod 5\% cream as primary or adjuvant therapy for melanoma in situ, lentigo maligna type. J Am Acad Dermatol 2015;72:1047-53.

18 Fan Q, Cohen S, John B, et al. Melanoma in situ treated with topical imiquimod for management of persistently positive margins: a review of treatment methods. Ochsner $\mathrm{J}$ 2015;15:443-7.

19 Smyth EC, Flavin M, Pulitzer MP, et al. Treatment of locally recurrent mucosal melanoma with topical imiquimod. $J$ Clin Oncol 2011;29:e809-11.

20 Spieth K, Kovács A, Wolter M, et al. Topical imiquimod: effectiveness in intraepithelial melanoma of oral mucosa. Lancet Oncol 2006; 7:1036-7.

21 Togashi Y, Shitara K, Nishikawa H. Regulatory T cells in cancer immunosuppression - implications for anticancer therapy. Nat Rev Clin Oncol 2019;16:356-71.

22 Okeke EB, Uzonna JE. The pivotal role of regulatory T cells in the regulation of innate immune cells. Front Immunol 2019;10:680. 